\title{
Squashing minimum coverings of 6-cycles into minimum coverings of triples
}

\author{
Elizabeth J. Billington, Selda Küçükçifçi, C.C. \\ Lindner and Mariusz Meszka
}

\begin{abstract}
A 6-cycle is said to be squashed if we identify a pair of opposite vertices and name one of them with the other (thereby turning the 6-cycle into a pair of triples with a common vertex). A 6-cycle can be squashed in six different ways. The spectrum for 6-cycle systems that can be squashed into Steiner triple systems has been determined by Lindner, Meszka and Rosa [From squashed 6-cycles to Steiner triple systems, J. Combin. Des. [6]]. The squashing of maximum packings of $K_{n}$ with 6-cycles into maximum packings of $K_{n}$ with triples has also been fully dealt with by Lindner, Lo Faro and Tripodi [Squashing maximum packings of 6-cycles into maximum packings of triples, submitted]. The object of this paper is to extend these results to minimum coverings of $K_{n}$ with 6-cycles into minimum coverings of $K_{n}$ with triples. We give a complete solution.
\end{abstract}

Mathematics Subject Classification (2010). Primary 05C38; Secondary 05B30, 05B40 .

Keywords. Steiner triple systems; Squashed 6-cycle systems; Minimum coverings.

\section{Introduction}

Recently three papers by Lindner et al. have dealt with so-called "squashing" of 6-cycles into triples ([6], [4]) and 8-cycles into 4-cycles ([5]). This paper completes the problem regarding the existence for all orders of squashable 6-cycle systems, by considering coverings.

So we begin with some definitions.

A Steiner triple system $\operatorname{STS}(n)$ of order $n$ (also called simply a "triple system") is a pair $(X, T)$ where $X$ is the vertex set of the complete graph $K_{n}$

The research of the fourth author was supported by the NCN Grant No. 2011/01/B/ST1/04056. 
and $T$ is a collection of edge-disjoint triangles (also called "triples") which partitions the edges of $K_{n}$.

A 6 -cycle system of order $n$ is a pair $(X, C)$ where $X$ is the vertex set of $K_{n}$ and $C$ is a collection of 6 -cycles which partitions the edge-set of $K_{n}$.

One connection between 6 -cycle systems and STS is given by 2-perfect 6-cycle systems; we omit details here, and refer interested readers to [7], or to the introduction in [6].

Here the connection between 6-cycles and triples is given by "squashing" a 6-cycle, which means identifying one pair of opposite vertices in the 6-cycle, forming a bowtie (that is, a pair of 3-cycles which share one vertex) from the 6-cycle. Each 6-cycle has three pairs of opposite vertices, and for each pair of opposite vertices there are two choices of vertex to remove in the "squashing"; so there are six possible bowties which can be formed from any one 6-cycle by squashing. See Figure 1, which illustrates this for the 6-cycle $(a, b, c, d, e, f)$.

If $B$ is any one of the six bowties in Figure 1, we say that we have squashed $(a, b, c, d, e, f)$ into $B$.

We note that the existence of bowtie systems for any admissible order was first determined by Horák and Rosa [2].

It is well-known that the spectrum (that is, the set of admissible orders) for 6 -cycle systems is all positive integers $n$ with $n \equiv 1$ or $9(\bmod 12)$ (see [8]), while the spectrum for $\operatorname{STS}(n)$ is the set of all positive integers $n$ with $n \equiv 1$ or $3(\bmod 6)([3])$. Thus the spectrum for which both a 6 -cycle system and an $\operatorname{STS}(n)$ exist is the set of all positive integers $n$ with $n \equiv 1$ or $9(\bmod$ 12).
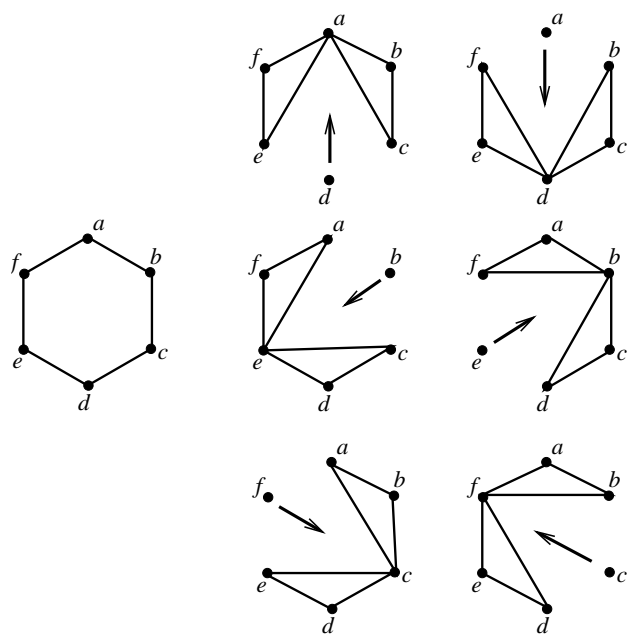

FiguRE 1

Let $K_{n}$ have vertex set $X$. Then a maximum packing $(X, C, L)$ of $K_{n}$ with 6-cycles is a decomposition of $E\left(K_{n}\right) \backslash L$ (the edges of $K_{n}$ with the edges 
in $L$ removed) into a maximum collection of cycles $C$, where the unused edges $L$ form a set as small as possible. Thus for instance if $n \equiv 1$ or $9(\bmod 12)$, then $L=\emptyset$. The set $L$ is called the leave.

In [6], a 6 -cycle system of every order $n \equiv 1$ or $9(\bmod 12)$ which can be squashed into an $\operatorname{STS}(n)$ was constructed. That paper also constructs a 6 -cycle maximum packing of order $n \equiv 3$ or $7(\bmod 12)$ which can be squashed into an $\operatorname{STS}(n)$. Subsequently, in [4], a 6-cycle maximum packing for every order $n$ (regardless of the congruency class of $n$ modulo 12), was constructed, which can be squashed into a maximum packing of $K_{n}$ with triples. The interested reader is referred to [4] for details. The object of this paper is the obvious problem of extending this result to minimum coverings of $K_{n}$ with 6-cycles. That is, we shall construct, for every possible order $n$ with $n>7$ a minimum covering of $K_{n}$ with 6 -cycles which can be squashed into a minimum covering of $K_{n}$ with triples.

We first need some more definitions.

Let $P \subseteq E\left(K_{n}\right)$ and let $C$ be a partition of $E\left(K_{n}\right) \cup P$ (where the union is strong, retaining repeated edges) into 6-cycles (or triples). If $|P|$ is as small as possible, then if the vertex set of $K_{n}$ is $X$, we say that $(X, C, P)$ is a minimum covering of $K_{n}$ with 6-cycles (or triples). Here $P$ is called the padding. (Several other papers have also used the term "excess" instead of "padding"; here we use the term padding.)

Table 1 lists some paddings for minimum coverings of $K_{n}$ with 6-cycles, and also with triples. In some cases, for 6-cycles, other paddings might be possible, on the same number of edges as the paddings given below; however, we use the simple paddings given in the table.

\begin{tabular}{|c|c|c|}
\hline Order $n$ & 6-cycles, padding & triples, padding \\
\hline \hline$n \equiv 0(\bmod 6)$ & 1 -factor & 1-factor \\
\hline$n \equiv 2,4(\bmod 6)$ & $T+\triangle$ & $T$ \\
\hline \hline$n \equiv 1,9(\bmod 12)$ & $\emptyset$ & $\emptyset$ \\
\hline \hline$n \equiv 3,7(\bmod 12)$ & $C_{3}$ & $\emptyset$ \\
\hline$n \equiv 5(\bmod 12)$ & $C_{5} \cup C_{3}$ & $C_{5}$ \\
\hline$n \equiv 11(\bmod 12)$ & $C_{5}$ & $C_{5}$ \\
\hline
\end{tabular}

Table 1: The paddings for 6-cycle coverings and for triple coverings of $K_{n}$ 
In Table 1, $T$ denotes a tripole, that is, a spanning subgraph of odd degree with precisely one vertex of degree 3 , and the rest of degree 1 . Also $T+\triangle$ denotes a spanning subgraph of odd degree with precisely four vertices of degree 3 (forming a $K_{4}$ ) and the rest of degree 1 . The padding denoted as $C_{5} \cup C_{3}$ is here taken to be two disjoint cycles of lengths 5 and 3 ; however, other paddings are possible.

Now let $(X, C, P)$ be a minimum covering of $K_{n}$ with 6 -cycles, where $P$ is one of the paddings given in Table 1 . Let $B$ be a collection of bowties obtained by squashing the 6 -cycles in $C$. If $P$ contains no triples (when $n \equiv$ $0,1,6,9$ or $11(\bmod 12))$, and $(X, B, P)$ is a minimum covering of $K_{n}$ with triples, or if $P$ contains a triple $b($ when $n \equiv 2,3,4,5,7,8$ or $10(\bmod 12))$, with $b \in B$ such that $(X, B \backslash b, P \backslash b)$ is a minimum covering of $K_{n}$ with triples, then we will say that we have squashed $(X, C, P)$ into a minimum covering of $K_{n}$ with triples.

We illustrate this definition with two examples.

Example 1.1. A minimum covering of $K_{11}$ with 6-cycles which squashes into a minimum covering of $K_{11}$ with triples.

$$
C=\{(2,3,4,5,0,1),(1,2,3,4,6,0), \quad(5,3,0,2,4,1), \quad(7,1,8,9,0,4),
$$

$(9,2,6,7,0,4),(8,2,5,7,10,0),(4,8,6,1,3,10),(9,3,8,5,10,1),(7,3,6,9,10,2)$, $(5,6,10,8,7,9)\}$. The padding $P$ is the 5 -cycle $(0,1,2,3,4)$.

Here the padding contains no triples and

$$
\begin{aligned}
B= & \{(2,3,4),(2,0,1),(1,2,3),(1,6,0),(5,3,0),(5,4,1),(7,1,8),(7,0,4), \\
& (9,2,6),(9,0,4),(8,2,5),(8,10,0),(4,8,6),(4,3,10),(9,3,8),(9,10,1), \\
& (7,3,6),(7,10,2),(5,6,10),(5,7,9)\} .
\end{aligned}
$$

It is straightforward to see that $(X, B, P)$ is a minimum covering of $K_{11}$ with triples.

Example 1.2. A minimum covering of $K_{8}$ with 6-cycles which squashes into a minimum covering of $K_{8}$ with triples.

$$
C=\{(2,3,4,5,0,1),(3,6,5,2,0,1),(3,0,2,4,7,1), \quad(6,7,0,3,2,1),
$$

$(5,7,2,6,0,4),(4,1,5,3,7,6)\}$. The padding $P$ is $K_{4}$ on $\{0,1,2,3\}$ together with the edges $\{4,5\},\{6,7\}$.

The padding here contains the triple $b=(0,1,2)$ which also belongs to $B$. Note that $(X, B \backslash\{(0,1,2)\}, P \backslash\{(0,1,2)\})$ is a minimum covering of $K_{8}$ with triples. Also $(P \backslash\{(0,1,2)\})$ is the tripole (see explanation below Table 1) with edges $\{\{3,0\},\{3,1\},\{3,2\},\{4,5\},\{6,7\}\}$.

\section{Preliminaries}

From now on, to say that the 6 -cycle $(a, b, c, d, e, f)$ is squashed we will always refer to the order in which the cycle is written, with first vertex $a$, and mean that it has been squashed into the bowtie $(a, b, c)(a, e, f)$; see Figure 2. 


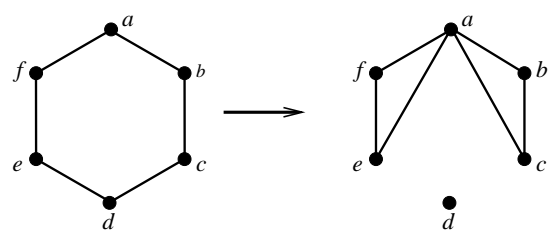

Figure 2

So, for example, in Example 1.1 above, we can simply list the 6-cycles in the given order (without listing the bowties they have been squashed into) and state that they can be squashed into a triple system. The triples can be deduced from the given 6-cycles with the stated 6-cycle orders.

The following examples are used frequently in the rest of the paper.

Example 2.1. A minimum covering of $K_{8} \backslash K_{4}$ with 6-cycles, which squashes into a minimum covering with triples.

The vertex set is $\{1,2,3,4,5,6,7,8\}$; the padding is the two edges 12 , 34 , and the hole of order 4 is $\{5,6,7,8\}$. The 6 -cycle covering which can be squashed into triples is:

$$
(1,5,2,3,4,8),(1,6,2,4,3,7),(3,5,4,1,2,8),(4,6,3,1,2,7) \text {. }
$$

Example 2.2. A minimum covering of $K_{10} \backslash K_{4}$ with 6-cycles, which squashes into a minimum covering with triples.

With vertex set $V\left(K_{10}\right)=\mathbb{Z}_{10}$, and hole $\{6,7,8,9\}$, the following 6 cycles squash into triples; the padding consists of the three disjoint edges $\{0,1\},\{2,3\},\{4,5\}$, which do not meet the hole.

$$
\begin{aligned}
& (6,1,0,7,3,2),(2,1,0,6,4,7),(3,5,4,2,0,8),(4,5,6,3,0,9),(7,1,3,4,0,5), \\
& (1,4,8,2,9,5),(2,3,9,1,8,5) .
\end{aligned}
$$

Subsequently we shall make frequent use of group divisible designs (GDDs) in our constructions. A 3-GDD of type $m^{x}$ consists of $m x$ points partitioned into $x$ groups of size $m$, with blocks (subsets) of size 3 satisfying the properties that every pair of points in different groups occurs in exactly one block, while pairs of points in the same group occur in no block. Similarly a 3-GDD of type $t^{1} m^{x}$ consists of $t+m x$ points partitioned into one group of size $t$ and $x$ groups of size $m$, with blocks of size 3 satisfying the same requirements regarding pairs of points and groups. See [1], Section IV.4.1 for results on existence of such GDDs. 
Elizabeth J. Billington, Selda Küçükçifçi, C.C. Lindner and Mariusz Meszka

\section{Even orders}

\subsection{Order $0(\bmod 6)$}

It is not possible to take a minimum covering of $K_{6}$ with 6 -cycles (that is, three 6-cycles and a 1-factor padding) which can be squashed into a 3-cycle minimum covering. This is shown in the following lemma.

Lemma 3.1. There is no minimum covering of $K_{6}$ with 6 -cycles which squashes into a minimum covering with triples.

Proof. Consider first a decomposition of $K_{6} \cup P$, where $P$ is a matching of $K_{6}$ with three edges, into three bowties $B_{1}, B_{2}$ and $B_{3}$. We let $V\left(K_{6}\right)=$ $\{1,2,3,4,5,6\}$.

Each vertex in $K_{6} \cup P$ has degree 6 , so if a vertex has degree 4 in one bowtie, it must have degree 2 in a second bowtie, and not occur at all in the third bowtie. So without loss of generality we assume that vertex 1 has degree 4 in $B_{1}$ and degree 2 in $B_{2}$; we assume that vertex 2 has degree 4 in $B_{2}$ and degree 2 in $B_{3}$; and we assume that vertex 3 has degree 4 in $B_{3}$ and degree 2 in $B_{1}$. Therefore edge $\{1,3\}$ is in $B_{1}$, edge $\{1,2\}$ is in $B_{2}$ and edge $\{2,3\}$ is in $B_{3}$. Since vertex 1 does not occur in $B_{3}$, vertex 2 does not occur in $B_{1}$ and vertex 3 does not occur in $B_{2}$, it is not possible for any of the edges $\{1,2\},\{2,3\},\{3,1\}$ to be in the matching $P$. So without loss of generality suppose that $P=\{\{1,4\},\{2,5\},\{3,6\}\}$. Therefore the edge $\{1,4\}$ lies in both $B_{1}$ and $B_{2}$, edge $\{2,5\}$ lies in $B_{2}$ and $B_{3}$, and edge $\{3,6\}$ lies in $B_{3}$ and $B_{1}$. We have now determined, without loss of generality, the three bowties:

$B_{1}=\{\{1,3,6\},\{1,4,5\}\}, B_{2}=\{\{2,1,4\},\{2,5,6\}\}$,

$B_{3}=\{\{3,2,5\},\{3,4,6\}\}$.

Now we need to transform each bowtie $B_{i}, i=1,2,3$, into a 6 -cycle $C_{i}$, $i=1,2,3$, to give a decomposition of $K_{6} \cup P$. Note that the edge $\{1,2\}$ may appear in either $C_{2}$ or $C_{3}$ (in the latter case vertex 1 is the vertex "removed" when the 6-cycle is pinched).

Suppose first that $\{1,2\} \in C_{2}$. Then cycle $C_{2}$ contains the path 2-1-4-3, while $C_{3}$ contains the path $3-2-5-1$. Thus $C_{1}$ must contain the edges $\{1,3\}$ and $\{2,4\}$, so $C_{1}$ is determined: $C_{1}=(1,3,6,2,4,5)$. But then the edge $\{1,5\}$ is in both $C_{1}$ and $C_{3}$, a contradiction, since we assumed that $\{1,5\} \notin P$.

The argument is similar if we assume instead that $\{1,2\} \in C_{3}$ : we find that 2-4-1-3 is a path in $C_{2}$ and 1-2-5-3 in $C_{3}$, so that edges $\{2,3\}$ and $\{1,5\}$ must be in $C_{1}$, so $C_{1}=(2,3,6,1,5,4)$ is forced. But now the edge $\{2,4\}$ is in both $C_{1}$ and $C_{2}$, a contradiction since $\{2,4\} \notin P$.

Therefore no 6 -covering of $K_{6}$ is possible which can be squashed into a 3 -covering.

Next we give some necessary small examples. 
Example 3.2. A minimum covering of $K_{12}$ with 6-cycles which squashes into a minimum covering with triples.

We take $V\left(K_{12}\right)=A \cup B \cup C$, where $A=\{1,2,3,4\}, B=\{5,6,7,8\}$ and $C=\{9,10,11,12\}$. Then we take three copies of Example 2.1 on the vertex sets $A \cup B, B \cup C$ and $C \cup A$, having holes (respectively) $B, C$ and $A$. The result is a suitable minimum covering of $K_{12}$ with 6 -cycles which can be squashed into triples.

Example 3.3. A minimum covering of $K_{12} \backslash K_{6}$ with 6-cycles which squashes into a minimum covering with triples.

Let $V\left(K_{12}\right)=\mathbb{Z}_{12}$; the padding consists of the three edges $\{0,1\},\{2,3\}$, $\{4,5\}$ and the hole is $\{6,7,8,9,10,11\}$; note that the padding does not meet the hole. The 6 -cycles which can be squashed are:

$(6,1,0,7,3,2),(7,1,0,6,3,2),(8,2,0,9,1,4),(9,3,0,8,1,5),(4,0,10,3,5,6)$, $(5,0,11,2,4,7),(2,1,11,5,4,9),(3,1,10,5,4,11),(5,2,10,4,3,8)$.

Example 3.4. A minimum covering of $K_{18}$ with 6-cycles which squashes into a minimum covering with triples.

Let $V\left(K_{18}\right)=A \cup B \cup C$ where $A, B, C$ are disjoint sets of size 6 . Place a copy of Example 3.3 on the sets $A \cup B, B \cup C$ and $C \cup A$, where in each case the hole is the second listed set. The result is a suitable 6 -cycle minimum covering which can be squashed into a 3-cycle minimum covering; the padding is a 1 -factor of $K_{18}$.

Example 3.5. A minimum covering of $K_{24}$ with 6-cycles which squashes into a minimum covering with triples.

We take $V\left(K_{24}\right)=\bigcup_{X=A, B, C}\left\{X_{1} \cup X_{2}\right\}$ where each $X_{1}, X_{2}$ for $X=A, B, C$ is of order 4 .

On the vertex set $X_{1} \cup X_{2}$ (for each $X=A, B, C$ ) we place a copy of $K_{8} \backslash K_{4}$ with hole $X_{1}$. (See Example 2.1.) Then on the holes, $A_{1} \cup B_{1} \cup C_{1}$, we place a copy of $K_{12}$ taken from Example 3.2. Finally, we use appropriate decompositions of $K_{4,4,4}$, three times, (see Example 9 in [6]) on each of the vertex sets $\left\{A_{1} \cup B_{2} \cup C_{2}\right\},\left\{B_{1} \cup A_{2} \cup C_{2}\right\},\left\{C_{1} \cup A_{2} \cup B_{2}\right\}$.

The result is a 6 -cycle minimum covering of $K_{24}$ which can be squashed into a minimum covering with triples; the padding is twelve disjoint edges (a 1-factor).

Example 3.6. A minimum covering of $K_{30}$ with 6-cycles which squashes into a minimum covering with triples.

Let $V\left(K_{30}\right)=X \cup P \cup Q$ where $X=\{25,26, \ldots, 30\}, P=\{1,2,3, \ldots, 12\}$ and $Q=\{13,14, \ldots, 24\}$. Now on the set $P$ we can take a 4 -GDD of type $3^{4}$, having four groups of size three, which we take as $\{1,2,3\},\{4,5,6\},\{7,8,9\}$, $\{10,11,12\}$, and blocks of size 4 . For each block $\{a, b, c, d\}$ in this 4-GDD, on the set $\{\{a, a+12\},\{b, b+12\},\{c, c+12\},\{d, d+12\}\}$, we take a copy of 
a 6-cycle packing of $K_{2,2,2,2}$ (which is the same as a 6-cycle packing of the graph $K_{8}$ minus a 1 -factor), which squashes into a 3 -cycle packing. Such an example is given in Example 2.2 of [4].

Then on the set $X \cup\{1,2,3,13,14,15\}$ we place a copy of Example 3.2, for $K_{12}$. Next, on $X \cup\{4,5,6,16,17,18\}$, on $X \cup\{7,8,9,19,20,21\}$ and on $X \cup\{10,11,12,22,23,24\}$, we place a copy of Example 3.3 , for $K_{12} \backslash K_{6}$, each time taking the hole of size 6 to be the vertices in $X$.

The result is a 6 -cycle minimum covering of $K_{30}$ which can be squashed into a 3-cycle minimum covering.

We are now ready to deal with the general case.

Lemma 3.7. Whenever $n \equiv 0(\bmod 6), n>6$, there is a minimum covering of $K_{n}$ by 6 -cycles which can be squashed into a minimum covering by triples.

Proof. First, when the order $n$ is $0(\bmod 12)$, we let $n=12 x$ and assume $x \geq 3$; the cases $x=1$ and $x=2$ appear in Examples 3.2 and 3.5 above.

We take $V\left(K_{12 x}\right)=\left\{v_{1}, v_{2} \mid v \in \mathbb{Z}_{6 x}\right\}$. On the set $\left\{v_{1} \mid v \in \mathbb{Z}_{6 x}\right\}$ we take a 3 -GDD of type $6^{x}$, since $x \geq 3$; see Section IV.4.1 of [1]. Then for each group of size 6 , say $\left\{a_{1}, b_{1}, c_{1}, d_{1}, e_{1}, f_{1}\right\}$, on the set $\left\{a_{1}, b_{1}, c_{1}, d_{1}, e_{1}, f_{1}\right\} \cup$ $\left\{a_{2}, b_{2}, c_{2}, d_{2}, e_{2}, f_{2}\right\}$ we place a copy of Example 3.2. Finally, for each block such as $\left\{p_{1}, q_{1}, r_{1}\right\}$ in the 3 -GDD, on the set $\left\{p_{1}, p_{2}\right\} \cup\left\{q_{1}, q_{2}\right\} \cup\left\{r_{1}, r_{2}\right\}$ we place a copy of a 6 -cycle decomposition of $K_{2,2,2}$ which squashes into a 3 -cycle decomposition; see Example 2.1 in [4], because of course $K_{2,2,2}$ is the same graph as $K_{6}$ minus a 1 -factor. This yields a minimum covering of $K_{12 x}$ by 6-cycles (with a 1-factor padding) which squashes into a minimum covering by triples.

Now consider $K_{12 x+6}$ where $x \geq 3$. The cases for orders 18 and 30 , when $x=1,2$, appear in Examples 3.4 and 3.6 above.

We let $V\left(K_{12 x+6}\right)=X \cup\left\{v_{1}, v_{2} \mid v \in \mathbb{Z}_{6 x+1}\right\}$, where $|X|=4$ and $x \geq 3$. On the set $\left\{v_{1} \mid v \in \mathbb{Z}_{6 x+1}\right\}$ we take a 3 -GDD of type $7^{1} 3^{2 x-2}$; this exists provided $x \geq 3$ (see Section IV.4.1 of [1]). Suppose the one group of size 7 is $\left\{0_{1}, 1_{1}, 2_{1}, 3_{1}, 4_{1}, 5_{1}, 6_{1}\right\}$. Then we take minimum coverings with 6 -cycles which squash into triples, as follows:

(1) On the set $X \cup\left\{v_{1}, v_{2} \mid 0 \leq v \leq 6\right\}$ we place a minimal covering of $K_{18}$ with 6 -cycles which can be squashed into a minimal covering by triples; see Example 3.4 above.

(2) For each group $\left\{a_{1}, b_{1}, c_{1}\right\}$ of size 3 in the 3 -GDD, on the set $X \cup$ $\left\{a_{i}, b_{i}, c_{i} \mid i=1,2\right\}$ we place a minimal covering of $K_{10} \backslash K_{4}$ with 6 cycles which can be squashed into a minimal covering by triples, with the set $X$ being the hole of size 4; see Example 2.2 above.

(3) For each block $\left\{p_{1}, q_{1}, r_{1}\right\}$ in the 3-GDD, on the set $\left\{p_{1}, p_{2}\right\} \cup\left\{q_{1}, q_{2}\right\} \cup$ $\left\{r_{1}, r_{2}\right\}$ we place a copy of a 6 -cycle decomposition of $K_{2,2,2}$ which squashes into a 3-cycle decomposition; see Example 2.1 in [4].

The result is a minimum covering of $K_{12 x+6}$ by 6 -cycles (with a 1 -factor padding) which squashes into a minimum covering by triples. 


\subsection{Orders 2 and $4(\bmod 6)$}

The case of order 8 is dealt with in Example 1.2 above. However, we also need the following examples here.

Example 3.8. A minimum covering of $K_{8} \backslash K_{2}$ with 6-cycles which squashes into a minimum covering of $K_{8}$ with triples.

With vertex set $\mathbb{Z}_{8}$, let the hole be $\{6,7\}$ and take the padding to be the disjoint edges (not meeting the hole): $\{0,1\},\{2,3\},\{4,5\}$. Then squashable 6-cycles are as follows:

$$
(2,3,4,5,0,1),(1,3,6,4,7,0),(5,3,0,2,4,1),(4,5,7,2,6,0),(2,3,7,1,6,5) .
$$

Example 3.9. A minimum covering of $K_{10}$ with 6-cycles which squashes into a minimum covering of $K_{10}$ with triples.

$$
C=\{(2,3,4,5,0,1),(2,3,5,4,0,1), \quad(5,6,0,2,4,1), \quad(4,8,0,2,5,7),
$$
$(3,0,9,7,6,1),(7,0,3,8,9,1),(8,1,3,6,9,2),(6,4,9,8,7,2),(8,5,9,3,7,6)\}$. The padding $P$ is $K_{4}$ on $\{0,1,2,3\}$ together with the edges $\{4,5\},\{6,7\},\{8,9\}$.

Example 3.10. A minimum covering of $K_{14}$ with 6-cycles which squashes into a minimum covering of $K_{14}$ with triples.

Let $V\left(K_{14}\right)=\mathbb{Z}_{14}$ and let $X=\{0,1,2,3,4,5\}$ and $Y=\{6,7, \ldots, 13\}$. On the set $Y$ we place a copy of a maximum packing of $K_{8}$ with 6 -cycles which squashes into a maximum packing with triples, with leave the set of edges $\{6,9\},\{7,8\},\{10,13\},\{11,12\}$; see Example 2.2 in [4] for this. Then on $X \backslash Y$, with padding $K_{4}$ on $\{0,1,2,3\}$ together with the edges $\{4,5\},\{6,7\}$, $\{8,9\},\{10,11\},\{12,13\}$, we place the following 6 -cycles which squash into triples:
$(3,2,1,0,10,5)$,
$(6,5,0,1,4,3)$,
$(1,2,0,3,10,11)$,
$(0,2,10,1,13,3)$,
$(0,4,12,1,3,11)$,
$(1,8,4,6,0,9)$,
$(7,1,6,2,8,0)$,
$(1,3,12,0,13,5)$,
$(7,2,3,9,5,4)$,
$(4,10,13,12,11,2)$,
$(2,5,8,7,6,9)$,
$(12,5,11,4,13,2)$,

$(9,8,3,7,5,4)$.

The result is a minimum covering of $K_{14}$ with 6 -cycles which squashes into a minimum covering of $K_{14}$ with triples.

Example 3.11. A minimum covering of $K_{26}$ with 6-cycles which squashes into a minimum covering of $K_{26}$ with triples.

Let $V\left(K_{26}\right)=\left\{\infty_{1}, \infty_{2}\right\} \cup\left\{x_{1}, x_{2} \mid x \in \mathbb{Z}_{12}\right\}$. Take a 3 -GDD on $\left\{x_{1} \mid\right.$ $\left.x \in \mathbb{Z}_{12}\right\}$ of type $3^{4}$. For one group, say $\left\{0_{1}, 1_{1}, 2_{1}\right\}$, on the set $\left\{\infty_{1}, \infty_{2}\right\} \cup$ $\left\{x_{1}, x_{2} \mid x=0,1,2\right\}$, we place a copy from Example 1.2 , of order 8 , with the $K_{4}$ part of the padding being on the points $\left\{\infty_{1}, \infty_{2}, 0_{1}, 0_{2}\right\}$. Then on the remaining three groups of size 3 in the GDD, say $\left\{a_{1}, b_{1}, c_{1}\right\}$, on the set $\left\{\infty_{1}, \infty_{2}\right\} \cup\left\{x_{1}, x_{2} \mid x=a, b, c\right\}$ we place a copy of Example 3.8, with the hole being $\left\{\infty_{1}, \infty_{2}\right\}$. Finally we use a decomposition of $K_{2,2,2}$ (as given in Example 2.1 in [4]) on the set $\left\{p_{1}, p_{2}\right\},\left\{q_{1}, q_{2}\right\},\left\{r_{1}, r_{2}\right\}$ for each block 
$\left\{p_{1}, q_{1}, r_{1}\right\}$ in the GDD. This completes a minimum covering of $K_{26}$ by 6 cycles which can be squashed into a minumum covering by triples.

Lemma 3.12. Whenever $n \equiv 2$ or $4(\bmod 6), n \geq 8$, there is a minimum covering of $K_{n}$ by 6-cycles which can be squashed into a minimum covering by triples.

Proof. We consider four cases according as the order is 2, 4, 8 or 10 (mod 12).

Order $4(\bmod 12)$

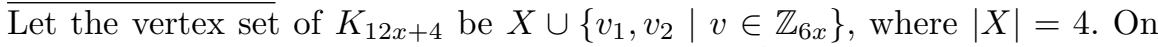
the set $\left\{v_{1} \mid v \in \mathbb{Z}_{6 x}\right\}$ we take a 3 -GDD of type $2^{3 x}$; this exists for all $x \geq 1$ (see Section IV.4.1 of [1]). Then a 6 -cycle minimum covering which can be squashed into a minimum covering of triples is obtained as follows:

(1) For one group of the 3-GDD, say $\left\{0_{1}, 1_{1}\right\}$, on the set $X \cup\left\{0_{1}, 1_{1}, 0_{2}, 1_{2}\right\}$ we place a copy of Example 1.2; the padding is $K_{4}$ with two disjoint edges, and we make sure that the two disjoint edges do not belong to $X$, while the $K_{4}$ padding is on the set $X$.

(2) For all other groups of the 3-GDD, say $\left\{a_{1}, b_{1}\right\}$, on the set $X \cup\left\{a_{1}, b_{1}, a_{2}, b_{2}\right\}$ we place a copy of Example 2.1, where $X$ is the hole of size 4 .

(3) For each triple such as $\left\{p_{1}, q_{1}, r_{1}\right\}$ in the 3 -GDD, on the set $\left\{p_{1}, p_{2}\right\} \cup$ $\left\{q_{1}, q_{2}\right\} \cup\left\{r_{1}, r_{2}\right\}$ we place an appropriate decomposition of $K_{2,2,2}$, as given in Example 2.1 of [4].

The result is a suitable minimum covering of $K_{12 x+4}$ by 6 -cycles which can be squashed into a minimum covering by triples; the padding is $T+\triangle$.

\section{Order $8(\bmod 12)$}

This case is exactly the same as the case $4(\bmod 12)$, except that $\mathbb{Z}_{6 x}$ is replaced by $\mathbb{Z}_{6 x+2}$, and a 3 -GDD of type $2^{3 x+1}$ is used, which also exists for all $x \geq 1$. The case of order 8 (when $x=0$ ) appears above in Example 1.2.

\section{Order $10(\bmod 12)$}

Consider $K_{12 x+10}$. Note that when $x=0$, the case for order 10 appears in Example 3.9 above. So we assume that $x \geq 1$. Let $V\left(K_{12 x+10}\right)=X \cup\left\{v_{1}, v_{2} \mid\right.$ $\left.v \in \mathbb{Z}_{6 x+3}\right\}$ where $|X|=4$. On the set $\left\{v_{1} \mid v \in \mathbb{Z}_{6 x+3}\right\}$ we take a Kirkman Triple System. (In fact a $3-G G G$ of type $3^{2 x+1}$ suffices.) Squashable minimum coverings by 6 -cycles are then taken as follows:

(1) If one group of the GDD is $\left\{0_{1}, 1_{1}, 2_{1}\right\}$, on the set $X \cup\left\{0_{1}, 1_{1}, 2_{1}, 0_{2}, 1_{2}, 2_{2}\right\}$ of size 10 we take a copy of Example 3.9.

(2) For all other groups of the GDD, such as, say $\left\{a_{1}, b_{1}, c_{1}\right\}$, on the set $X \cup\left\{a_{1}, b_{1}, c_{1}, a_{2}, b_{2}, c_{2}\right\}$ we place a copy of Example 2.2, ensuring that the hole is the set $X$ of size 4 .

(3) For each triple such as $\left\{p_{1}, q_{1}, r_{1}\right\}$ in the 3-GDD, on the set $\left\{p_{1}, p_{2}\right\} \cup$ $\left\{q_{1}, q_{2}\right\} \cup\left\{r_{1}, r_{2}\right\}$ we place an appropriate decomposition of $K_{2,2,2}$, as given in Example 2.1 of [4]. 
Since all the above 6-cycles are squashable into minimum coverings of triples, this completes the case $10(\bmod 12)$.

Order $2(\bmod 12)$

Consider $K_{12 x+2}$. When $x=1$, the order is 14 , and this case appears in Example 3.10 above. The case for $x=2$ (order 26) is dealt with above in Example 3.11. So henceforth we assume that $x \geq 3$. We take $V\left(K_{12 x+2}\right)=$ $X \cup\left\{v_{1}, v_{2} \mid v \in \mathbb{Z}_{6 x-1}\right\}$ where $|X|=4$. On the set $\left\{v_{1} \mid v \in \mathbb{Z}_{6 x-1}\right\}$ we take a 3 -GDD of type $5^{1} 3^{2 x-2}$ which exists for $x \geq 3$. Squashable minimum coverings by 6 -cycles are then taken as follows:

(1) Suppose the group of size 5 in the 3 -GDD is $\left\{0_{1}, 1_{1}, 2_{1}, 3_{1}, 4_{1}\right\}$. Then on the set $X \cup\left\{v_{1}, v_{2} \mid v=0,1,2,3,4\right\}$, we place a copy of Example 3.10.

(2) We repeat step (2) (for all groups of size 3 in this 3 -GDD) in the above case of order $10(\bmod 12)$.

(3) We repeat step (3) in the above case of order $10(\bmod 12)$.

The above four cases (modulo 12) complete the lemma.

\section{Odd orders}

When the order of the complete graph is 1 or $9(\bmod 12)$, there is an exact 6-cycle system which can be squashed into a Steiner triple system; see [6]. So in this section we deal with the remaining odd orders (modulo 12), for which the padding is non-empty.

\subsection{Orders 3 and $7(\bmod 12)$}

An exhaustive computer search has shown that it is not possible to find a minimum covering of $K_{7}$ (with the padding being one triangle) with 6-cycles which can be squashed into an STS(7) once the triangle padding is removed.

We now give some necessary small examples.

Example 4.1. A minimum covering of $K_{3,3,3}$ plus a padding of one 3-cycle, by 6 -cycles, which squashes into a minimum covering of $K_{3,3,3}$ with triples.

With vertex set $\{0,1,2\} \cup\{3,4,5\} \cup\{6,7,8\}$, and padding the 3 -cycle $(0,3,6)$, the following 6 -cycles give a covering which squashes into a 3 -cycle covering.

$$
(6,0,3,1,4,2),(3,2,7,5,8,0),(7,0,4,6,5,1),(5,2,8,3,6,0),(1,6,3,7,4,8) .
$$


Example 4.2. A minimum covering of $K_{15}$ with 6-cycles which squashes into a minimum covering of $K_{15}$ with triples; the padding is one 3-cycle.

With vertex set $\mathbb{Z}_{15}$ and padding the 3 -cycle $(0,1,2)$, the following 6 cycles give a covering which squashes into an $\operatorname{STS}(15)$ upon removal of the triangle padding.

$\begin{array}{llll}(1,0,3,10,5,2), & (0,1,2,13,10,6), & (4,0,2,7,9,1), & (11,5,0,2,14,12), \\ (7,0,9,4,2,6), & (14,0,8,12,2,9), & (12,13,0,10,1,6), & (8,2,11,0,12,9), \\ (14,7,3,1,5,6), & (1,8,10,14,11,7), & (13,14,1,11,8,7), & (2,10,12,1,13,3), \\ (4,3,11,9,5,8), & (3,5,12,4,6,8), & (4,13,6,3,12,7), & (10,9,3,14,5,7), \\ (9,13,5,4,11,6), & (10,4,14,8,13,11) . & & \end{array}$

Example 4.3. A minimum covering of $K_{19}$ with 6-cycles which squashes into a minimum covering of $K_{19}$ with triples; the padding is one 3-cycle.

We take the vertex set $\left\{i_{j} \mid 1 \leq i \leq 6, j=1,2,3\right\} \cup\{\infty\}$.

(1) On the three sets $\left\{i_{j},(i+1)_{j} \mid j=1,2,3\right\} \cup\{\infty\}$, for $i=1,3,5$, place a maximum packing of $K_{7}$ with 6 -cycles which can be squashed into triples, with leave $\left\{(i+1)_{1},(i+1)_{2},(i+1)_{3}\right\}$, in each case (so $i+1=2,4,6)$. (See Example 4 in [6].) This yields nine 6 -cycles.

(2) On the set $\left\{2_{j}, 4_{j}, 6_{j} \mid j=1,2,3\right\}$, of size 9 , place a 6 -cycle system of $K_{9}$ which can be squashed into triples (see Example 1 in [6]); this is six 6-cycles, squashed into 12 triples.

(3) For (3), (4) and (5), note that one $\operatorname{STS}(7)$ on $\{1,2,3,4,5,6, \infty\}$ is given by the triples $\{\infty 12, \infty 34, \infty 56,246,235,136,145\}$.

On the set $\left\{2_{1}, 2_{2}, 2_{3}\right\} \cup\left\{3_{1}, 3_{2}, 3_{3}\right\} \cup\left\{5_{1}, 5_{2}, 5_{3}\right\}$ place a copy of the example in Lemma 11 of $[6]\left(K_{3,3,3} \backslash K_{3}\right)$, with hole $\left\{2_{1}, 3_{1}, 5_{1}\right\}$. This contains four 6-cycles, squashable into eight triples.

(4) Repeat (3) for $\left\{1_{1}, 3_{1}, 6_{1}\right\}$ instead of $\left\{2_{1}, 3_{1}, 5_{1}\right\}$.

(5) Repeat (3) for $\left\{1_{1}, 4_{1}, 5_{1}\right\}$ instead of $\left\{2_{1}, 3_{1}, 5_{1}\right\}$.

(6) So far we have used all edges of $K_{19}$ except for the triples $\left\{2_{1}, 3_{1}, 5_{1}\right\}$, $\left\{1_{1}, 3_{1}, 6_{1}\right\}$,

$\left\{1_{1}, 4_{1}, 5_{1}\right\}$. We now add in the padding $\left\{2_{1}, 4_{1}, 6_{1}\right\}$. So we take a maximum packing of $K_{2,2,2}$ on the vertex set $\left\{1_{1}, 2_{1}\right\} \cup\left\{3_{1}, 4_{1}\right\} \cup\left\{5_{1}, 6_{1}\right\}$, with two 6-cycles which squash into triples:

$$
\left(6_{1}, 1_{1}, 3_{1}, 5_{1}, 4_{1}, 2_{1}\right),\left(5_{1}, 2_{1}, 3_{1}, 6_{1}, 4_{1}, 1_{1}\right) .
$$

We now have $K_{19}$ plus the padding $\left\{2_{1}, 4_{1}, 6_{1}\right\}$ decomposed into 296 cycles which can be squashed into 58 triples, one of which is $\left\{2_{1}, 4_{1}, 6_{1}\right\}$. Discarding this triple leaves an STS(19) on 57 triples, as required.

Example 4.4. A minimum covering of $K_{27}$ with 6-cycles which squashes into a minimum covering of $K_{27}$ with triples; the padding is one 3-cycle.

We take the vertex set $\left\{i_{j} \mid 1 \leq i \leq 9, j=1,2,3\right\}$. Then 6 -cycles (which squash into triples) are taken as follows: 
(1) On the sets $\left\{1_{j}, 2_{j}, 3_{j} \mid j=1,2,3\right\},\left\{4_{j}, 5_{j}, 6_{j} \mid j=1,2,3\right\}$ and $\left\{7_{j}, 8_{j}, 9_{j} \mid j=1,2,3\right\}$ we place squashable 6 -cycle systems of order 9. (We also use a Kirkman triple system $\operatorname{KTS}(9)$ on $\{i \mid 1 \leq i \leq 9\}$, with one parallel class $\{123,456,789\}$.)

(2) There are nine more triples in the $\operatorname{KTS}(9)$ besides $\{123,456,789\}$. For each of these nine triples, say $\{x y z\}$, on the set $\left\{x_{j} \mid j=1,2,3\right\} \cup$ $\left\{y_{j} \mid j=1,2,3\right\} \cup\left\{z_{j} \mid j=1,2,3\right\}$, we place a maximum packing of $K_{3,3,3}$ with 6 -cycles, with leave $\left\{x_{1}, y_{1}, z_{1}\right\}$, which can be squashed into triples (see Lemma 11 of [6]).

(3) The edges of $K_{27}$ which are so far unused are those in $K_{3,3,3}$ on the vertex set $\left\{1_{1}, 2_{1}, 3_{1}\right\} \cup\left\{4_{1}, 5_{1}, 6_{1}\right\} \cup\left\{7_{1}, 8_{1}, 9_{1}\right\}$. We include the padding $\left\{1_{1}, 4_{1}, 7_{1}\right\}$, and place a copy of Example $4.1\left(K_{3,3,3}\right.$ plus a triangle) on here.

This completes the case of order 27 .

Example 4.5. A minimum covering of $K_{31}$ with 6-cycles which squashes into a minimum covering of $K_{31}$ with triples; the padding is one 3-cycle.

Let $V\left(K_{31}\right)=X \cup\left\{x_{1}, x_{2} \mid x \in \mathbb{Z}_{14}\right\}$, where $|X|=3$. There exists a 3 -GDD of type $6^{1} 2^{4}$ on 14 points, so we use decompositions of $K_{15}$ (Example 4.2), with the group of size 6 , and $K_{7} \backslash K_{3}$ (see Example 4 in [6]) with the four groups of size 2 , and then $K_{2,2,2}$ as usual with each block of the GDD. $12)$.

We can now deal with the general case when the order is 3 or $7(\bmod$

Lemma 4.6. Whenever $n \equiv 3$ or $7(\bmod 12), n \geq 15$, there is a minimum covering of $K_{n}$ by 6-cycles which can be squashed into a minimum covering by triples.

Proof. For $K_{12 x+3}$, note that $12 x+3=3+2(6+2(3 x-3))$. Our construction requires $x \geq 3$ in order to use a 3 -GDD of type $6^{1} 2^{3 x-3}$. When $x=1$, Example 4.2 provides a solution, and when $x=2$, Example 4.4 provides a solution. For order $12 x+3$ when $x \geq 3$, take the vertex set $\left\{i_{j} \mid 1 \leq\right.$ $i \leq 6 x, j=1,2\} \cup\left\{\infty_{1}, \infty_{2}, \infty_{3}\right\}$. Let the groups of a 3 -GDD of type $6^{1} 2^{3 x-3}$ be $\{1,2,3,4,5,6\},\{7,8\}, \ldots,\{6 x-1,6 x\}$. On the set $\left\{i_{j} \mid 1 \leq i \leq\right.$ $6, j=1,2\} \cup\left\{\infty_{1}, \infty_{2}, \infty_{3}\right\}$ we place a copy of Example 4.2, with padding $\left\{\infty_{1}, \infty_{2}, \infty_{3}\right\}$. Then for each group of the GDD $\{a, a+1\}$ of size 2 , on the set $\left\{a_{1}, a_{2},(a+1)_{1},(a+1)_{2}, \infty_{1}, \infty_{2}, \infty_{3}\right\}$ we place a squashable 6 -cycle maximum packing of $K_{7}$ (Example 4 in [6]) with leave $\left\{\infty_{1}, \infty_{2}, \infty_{3}\right\}$. Finally for each block, say $p q r$ of the 3-GDD, on the set $\left\{p_{1}, p_{2}\right\} \cup\left\{q_{1}, q_{2}\right\} \cup\left\{r_{1}, r_{2}\right\}$ we place a copy of a squashable 6 -cycle decomposition of $K_{2,2,2}$ (see Example 2.1 in [4]).

Similarly, for $K_{12 x+7}$, we note that $12 x+7=3+2(8+2(3 x-3))$, and we use a 3 -GDD of type $8^{1} 2^{3 x-3}$, which exists provided $x \geq 3$. When $x=0$, order 7 cannot be done; for $x=1$ (order 19), see Example 4.3; and for $x=2$ 
E4izabeth J. Billington, Selda Küçükçifçi, C.C. Lindner and Mariusz Meszka

(order 31), see Example 4.5. For the general case, with $x \geq 3$, as in the case $3(\bmod 12)$ we use Example 4.3 once (instead of order 15 as in the case 3 $(\bmod 12))$, and then the rest proceeds exactly as in the case $3(\bmod 12)$.

\subsection{Order $5(\bmod 12)$}

We begin with an example of order 9 having two disjoint holes of size 3 .

Example 4.7. A decomposition of $K_{9}$ with two disjoint holes of size 3, into 6-cycles which squash into a triple decomposition.

We take the vertex set $\mathbb{Z}_{9}$, with the two disjoint holes $\{0,1,2\}$ and $\{3,4,5\}$. The squashable 6 -cycles are as follows:

$$
(4,7,1,3,0,8),(3,8,1,4,0,6),(5,0,7,2,6,1),(2,3,7,6,5,8),(6,4,2,5,7,8) \text {. }
$$

Example 4.8. A minimum covering of $K_{17}$ with 6-cycles which squashes into a minimum covering of $K_{17}$ with triples; the padding is a 5-cycle and a 3-cycle.

We take the vertex set $\mathbb{Z}_{17}$, and padding the 3 -cycle $(0,1,2)$ and 5-cycle $(3,4,5,6,7)$. The following 6 -cycles squash into triples, and leave a hole on the vertices $\{8,9,10,11,12,13,14,15,16\} \backslash\{(8,9,10),(12,13,14)\}$. By filling this hole with a system of order 9 having two disjoint holes of size 3 (see Example 4.7), we complete the example of order 17.

$$
\begin{array}{llll}
(0,1,2,4,6,11), & (2,12,1,0,13,3), & (8,2,0,10,9,1), & (4,11,2,0,6,10), \\
(0,3,15,2,5,12), & (9,4,0,8,10,2), & (2,16,5,0,15,7), & (10,7,0,9,8,5), \\
(0,14,13,5,1,16), & (5,4,13,2,1,14), & (7,5,4,3,1,11), & (15,1,4,16,6,5), \\
(13,1,6,14,7,12), & (3,8,4,7,1,10), & (14,2,6,13,7,3), & (16,3,4,12,6,7), \\
(12,3,6,15,4,14), & (7,8,6,5,3,9), & (5,9,6,7,3,11) . &
\end{array}
$$

Example 4.9. A minimum covering of $K_{5,5,5} \backslash K_{3}$ with 6 -cycles which squashes into a triple decomposition minus one $K_{3}$.

We take the vertex set $\{0,1,2,3,4\} \cup\{5,6,7,8,9\} \cup\{10,11,12,13,14\}$, and remove the triangle $\{0,5,10\}$. The following 6 -cycles squash into a triple decomposition (missing the triple $\{0,5,10\}$ ).

$$
\begin{array}{llll}
(9,4,14,8,0,13), & (8,3,14,9,0,12), & (7,0,11,9,2,14), & (6,0,14,5,4,13), \\
(10,1,8,13,3,6), & (9,1,12,7,2,10), & (4,10,7,1,11,8), & (1,6,11,4,7,13), \\
(5,1,14,6,4,12), & (13,2,8,10,3,5), & (12,6,2,11,7,3), & (11,5,2,12,9,3) .
\end{array}
$$


Example 4.10. A minimum covering of $K_{17} \backslash K_{5}$ with 6-cycles which squashes into a minimum covering of $K_{17} \backslash K_{5}$ with triples.

We take the vertex set to be $\left\{i_{j} \mid 1 \leq i \leq 5, j=1,2,3\right\} \cup\left\{\infty_{1}, \infty_{2}\right\}$. The hole of size 5 will be $\left\{1_{1}, 1_{2}, 1_{3}, \infty_{1}, \infty_{2}\right\}$. On the three sets $\left\{1_{j}, 2_{j}, 3_{j}, 4_{j}, 5_{j}\right.$, $\left.\infty_{1}, \infty_{2}\right\}$, for $j=1,2,3$, we place a maximum packing of $K_{7}$ with 6 -cycles which can be squashed into triples (see Example 4 in [6]) with leave $\left\{1_{j}, \infty_{1}, \infty_{2}\right\}$ for each $j$. Then on the sets $\left\{i_{1} \mid i \leq 5\right\} \cup\left\{i_{2} \mid i \leq 5\right\} \cup\left\{i_{3} \mid i \leq 5\right\}$ we place a copy of Example 4.9 where the leave is the set $\left\{1_{1}, 1_{2}, 1_{3}\right\}$. This completes the example.

The following example is needed to complete the case of order 29 .

Example 4.11. A covering of $K_{11} \backslash\left(K_{5} \cup K_{3}\right)$, where the holes of size 5 and 3 share one vertex, with 6-cycles, which squashes into a collection of triples.

We take the vertex set $\mathbb{Z}_{11}$, where the holes of size 3 and 5 are $\{0,1,2\} \cup$ $\{2,3,4,5,6\}$ (the holes share one common vertex).

The following 6-cycles squash into a triple decomposition:

$$
\begin{array}{llll}
(7,5,0,3,1,4), & (0,4,8,2,9,6), & (3,9,1,7,0,10), & (2,7,9,0,8,10), \\
(1,5,10,7,8,6), & (9,4,10,1,8,5), & (7,6,10,9,8,3) . &
\end{array}
$$

Example 4.12. A minimum covering of $K_{29}$ with 6-cycles which squashes into a minimum covering of $K_{29}$ with triples; the padding is a 5-cycle and a 3-cycle.

We take the vertex set $\left\{i_{j} \mid 1 \leq i \leq 8, j=1,2,3\right\} \cup X$, where $X=$ $\left\{\infty_{1}, \infty_{2}, \infty_{3}, \infty_{4}, \infty_{5}\right\}$.

(1) On the set $X \cup\left\{1_{j}, 2_{j} \mid j=1,2,3\right\}$, we place a copy of Example 1.1, which has ten 6 -cycles and padding a 5-cycle (which we ensure lies entirely within the set $X)$.

(2) On the three sets $X \cup\left\{3_{j}, 4_{j} \mid j=1,2,3\right\}, X \cup\left\{5_{j}, 6_{j} \mid j=1,2,3\right\}$, and $X \cup\left\{7_{j}, 8_{j} \mid j=1,2,3\right\}$, we place a copy of Example 4.11, of $K_{11}$ with holes of size 5 (on $X$ ) and size 3 (being $\left\{\infty_{5}, 3_{1}, 4_{1}\right\},\left\{\infty_{5}, 5_{1}, 6_{1}\right\}$, $\left\{\infty_{5}, 7_{1}, 8_{1}\right\}$, respectively).

(3) On the set $\left\{\infty_{5}, 1_{1}, 2_{1}, \ldots, 7_{1}, 8_{1}\right\}=Y$, we take an $\operatorname{STS}(9)$ containing the triples $\left\{\infty_{5}, 1_{1}, 2_{1}\right\},\left\{\infty_{5}, 3_{1}, 4_{1}\right\},\left\{\infty_{5}, 5_{1}, 6_{1}\right\},\left\{\infty_{5}, 7_{1}, 8_{1}\right\}$.

(4) For each of the eight triples not involving $\infty_{5}$ in the $\operatorname{STS}(9)$ in (3), such as, say $\left\{x_{1}, y_{1}, z_{1}\right\}$, on the sets $\left\{x_{1}, x_{2}, x_{3}\right\} \cup\left\{y_{1}, y_{2}, y_{3}\right\} \cup\left\{z_{1}, z_{2}, z_{3}\right\}$ we place a decomposition of $K_{3,3,3} \backslash\left\{x_{1}, y_{1}, z_{1}\right\}$ into four squashable 6-cycles (see Lemma 11 in [6]).

(5) All edges in $K_{29}$ have now been covered except for those in $Y \backslash\left\{\infty_{5}, 1_{1}, 2_{1}\right\}$ (see $(3))$. We now adjoin the padding $\left\{\infty_{5}, 1_{1}, 2_{1}\right\}$. Then $Y$ can be partitioned into six squashable 6 -cycles (see Example 1 in [6]) so that $\left\{\infty_{5}, 1_{1}, 2_{1}\right\}$ is one of the triples from the squashing. 
(6) Now removing the triple $\left\{\infty_{5}, 1_{1}, 2_{1}\right\}$ gives a minimum covering of $K_{29}$ with triples from the squashed 6 -cycles, with padding the 5 -cycles inside $X$.

Lemma 4.13. Whenever $n \equiv 5(\bmod 12), n \geq 17$, there is a minimum covering of $K_{n}$ by 6-cycles which can be squashed into a minimum covering by triples.

Proof. Order 17 is covered by Example 4.8, and order 29 by Example 4.12. So consider $K_{12 x+5}$ where $x \geq 3$. For order $12 x+5$, we take the vertex set

$$
\left\{i_{j} \mid 1 \leq i \leq 6 x, j=1,2\right\} \cup\left\{\infty_{k} \mid k=1,2,3,4,5\right\} .
$$

We use a 3 -GDD of type $6^{x}$ (which exists for $x \geq 3$; see [1]) on the set $\{1,2, \ldots, 6 x\}$, with groups $\{6 a+1,6 a+2, \ldots, 6 a+6\}$ for $a=0,1, \ldots, x-1$. Then we use Example 4.8 once, on the set $\left\{i_{j} \mid 1 \leq i \leq 6, j=1,2\right\} \cup\left\{\infty_{k} \mid\right.$ $1 \leq k \leq 5\}$; Example 4.10, $x-1$ times, on $\left\{(6 a+i)_{j} \mid 1 \leq i \leq 6, j=\right.$ $1,2\} \cup\left\{\infty_{k} \mid 1 \leq k \leq 5\right\}$, for $a=1,2, \ldots, x-1$, with the obvious hole of size 5 ; and for each triple $p q r$ of the 3 -GDD, on the sets $\left\{p_{1}, p_{2}\right\} \cup\left\{q_{1}, q_{2}\right\} \cup\left\{r_{1}, r_{2}\right\}$, we use our squashable 6-cycle decomposition of $K_{2,2,2}$ (see Example 2.1 in $[4])$.

\subsection{Order $11(\bmod 12)$}

Recall that the case of order 11 was dealt with earlier in Example 1.1. No further examples are required for orders $11(\bmod 12)$. We have the following result.

Lemma 4.14. Whenever $n \equiv 11(\bmod 12), n \geq 11$, there is a minimum covering of $K_{n}$ by 6-cycles which can be squashed into a minimum covering by triples.

Proof. The case of order 11 appears in Example 1.1, so we consider $K_{12 x+11}$ where $x>0$. We let the vertex set be $X \cup\left\{v_{1}, v_{2} \mid v \in \mathbb{Z}_{6 x+4}\right\}$ where $|X|=3$, and we use a 3-GDD of type $4^{1} 2^{3 x}$, which exists for $x>0$. Let the group of size 4 be $\left\{0_{1}, 1_{1}, 2_{1}, 3_{1}\right\}$. The "ingredients" are then (i) Example 1.1 on the set $X \cup\left\{0_{1}, 1_{1}, 2_{1}, 3_{1}, 0_{2}, 1_{2}, 2_{2}, 3_{2}\right\}$ (with its padding $C_{5}$ not meeting the set $X$ ); (ii) a maximum packing of order 7 with leave the set $X$ on the sets $X \cup\left\{a_{1}, b_{1}, a_{2}, b_{2}\right\}$ for each group $\left\{a_{1}, b_{1}\right\}$ in the GDD; and (iii) copies of $K_{2,2,2}$, using each block of size 3 from the GDD in the usual way.

The result is a suitable minimum covering of $K_{12 x+11}$ by 6 -cycles, with padding a 5-cycle, which can be squashed into a minimum covering by triples.

\section{Concluding comments}

Combining results from [6] with the Lemmas 3.7, 3.12, 4.6, 4.13, and 4.14 above, we have now shown the following. 
Theorem 5.1. For each order $n \geq 8$, there is a minimum covering of $K_{n}$ with 6-cycles which can be squashed into a minimum covering of $K_{n}$ with triples.

\section{References}

[1] Colbourn, C.J., Dinitz, J.H. (eds.): The CRC Handbook of Combinatorial Designs. Second Edition, Chapman and Hall/CRC, Boca Raton, FL (2007).

[2] Horák, P., Rosa, A.: Decomposing Steiner triple systems into small configurations. Ars Combin. 26, 91-105 (1988).

[3] Kirkman, T.P.: On a problem in combinations. Cambridge and Dublin Math. J. 2, 191-204 (1847).

[4] Lindner, C.C., Lo Faro, G., Tripodi, A.: Squashing maximum packings of 6cycles into maximum packings of triples, (submitted).

[5] Lindner, C.C., Lo Faro, G., Meszka, M., Tripodi, A.: Squashing maximum packings of $K_{n}$ with 8-cycles into maximum packings of $K_{n}$ with 4-cycles, Filomat (to appear).

[6] Lindner, C.C., Meszka, M., Rosa, A.: From squashed 6-cycles to Steiner triple systems. J. Combin. Des. 22, 189-195 (2014).

[7] Lindner, C.C., Phelps, K.T., Rodger, C.A.: The spectrum for 2-perfect 6-cycle systems. J. Combin. Theory Ser. A 57, 76-85 (1991).

[8] Rosa, A. : On cycle decompositions of the complete graph into $(4 m+2)$-gons. Mat-fyz Casopis. 16, 349-435 (1966).

Elizabeth J. Billington

School of Mathematics and Physics

The University of Queensland

Brisbane, QLD 4072, Australia

e-mail: ejb@maths.uq.edu .au

Selda Küçükçifçi

Department of Mathematics, Koç University

Rumelifeneri Yolu, 34450, Sarıer

Istanbul, Turkey

e-mail: skucukcifci@ku.edu.tr

C.C. Lindner

Department of Mathematics and Statistics

Auburn University

Auburn, AL 36849-5307, U.S.A.

e-mail: lindncc@auburn.edu

Mariusz Meszka

AGH University of Science and Technology

Kraków, 30059 Poland

e-mail: meszka@agh.edu.pl 